\title{
ANTROPOLOGÍA DE LA SALUD: PATOCENOSIS COMPARADAS... ALGO SE NOS ESCAPA
}

\author{
Lic. D. ${ }^{a} M .{ }^{a}$ Teresa Miret Garcia \\ Universidad Católica de Murcia. España \\ Lic. D. Alvaro Bernalte Benazet \\ Universidad de Cádiz. España \\ Alexandre Bernalte y Nuria López
}

\section{INTRODUCCIÓN}

En lo que podíamos llamar la vorágine del día a día en la docencia universitaria, quizás no se nos otorgan muchas oportunidades para, haciendo un alto en el camino, reflexionar sobre nuestras estrategias docentes, nuestro discurso,... o bajando un poco el listón, sobre la orientación o dirección hacia el que ,muchas veces, orienta o lleva el mismo, a los estudiantes.

Cuando nos permitimos esa reflexión, y se produce entre personas con otra formación de base, con otras funciones dentro de la universidad, y con vivencias en diferentes puntos de la geografia - a veces y sólo a veces- emerge una llamada de atención, sobre nuestro enfoque pedagógico y sobre el cumplimiento de nuestros objetivos generales, que en nuestro caso reconocemos como dos:

$1^{0}$. Transmitir información o compartirla, y preparar al alumno para que, si no la tiene, sepa donde buscarla.

$2^{\circ}$. Fomentar un espíritu crítico en los estudiantes.

Esta constatación de que "algo no está bien", surgió de un debate interesante sobre la enseñanza clínica en Medicina, donde, curiosamente, se transmiten una serie de categorías mórbidas $^{1}$, y partiendo de ese rótulo "enfermedad tal", se describen: la clínica, la etiología,..., el tratamiento y el pronóstico; curiosamente después de años de estructurar el pensamiento en esa dirección, los estudiantes se dan cuenta de que van a trabajar exactamente a la inversa, es decir, que las personas no llevan un cartel anunciador de la enfermedad que

1 NOTA DE LOS AUTORES. Podemos denominarlas también enfermedades, patologías y síndromes. 
les aflige, sino que sólo demuestran su sufrimiento (lo cual es, en algunos casos, tomado casi como una información banal ${ }^{2}$ ) y a partir de ahí, de ese momento hay que recorrer el camino inverso al aprendido para tratar de rotular (diagnosticar) lo que les pasa a estas personas que acuden a la consulta.

A diferencia de los clínicos -quizás por el aura de provenir de la Salud Pública y de la Medicina Preventiva y Social - los salubristas, nos creemos sí afectos de otros pecados pero no de éste, que a nivel metafórico, sería enseñar los árboles -enfermedades - uno a uno de tal forma que no se vea el bosque, que serían una serie de porqués: existen esas enfermedades en unas poblaciones si, y en otras no; que perfiles de enfermedad tienen ciertas poblaciones debido a su cultura, su desarrollo socioeconómico, etc.

Es cierto que, tanto en la Epidemiología Clásica como en la Social, en la Salud Pública y la Medicina Preventiva y Social hablamos de problemas "generales" de estos hechos vitales como son el enfermar y el morir; pero no es menos cierto que hablamos de las entidades mórbidas una a una y por pequeños agrupamientos, por ejemplo según mecanismos de transmisión ${ }^{3}$, grupos de agentes causales, etc.

Este discurso y su orientación (descripción, enfermedad a enfermedad) impiden hacer notorias las desigualdades en materia de acceso a la salud y al tratamiento de la enfermedad de diversas poblaciones (bosque), es pues - como habrán podido comprobar a estas alturas del texto- , esta reflexión, un "mea culpa" sobre nuestra forma de abordar pedagogicamente una asignatura -la Salud Pública-, dado que, a lo largo de la Historia de esta disciplina ha habido suficientes aldabonazos en el sentido de nuestra autocrítica, que si bien nos llamaron la atención como referencias de un buen hacer en su momento, parece que las hemos olvidado ${ }^{4}$ dentro de esa esquizofrenia tan común entre la teorización y la práctica diaria.

Por último, mencionar que esta reflexión está muy en la línea de lo que es el trabajo de los antropólogos, y en particular de los que nos dedicamos al complejo salud - enfermedad - sistema de cuidados ${ }^{5}$, de problematizar la realidad; en definitiva, nuestra pretensión (objetivo) ha sido establecer cómo abordar la enseñanza de la Salud Pública y otras disciplinas afines, partiendo de la reflexión de conjunto que nos puede dar el conocimiento de la patocenosis $^{6}$ de unas serie de zonas de lo que denominamos mundo, al objeto de que su contrastación nos permitiera ver esas "desigualdades o diferencias" que, al cabo del tiempo,

2 NOTA DE LOS AUTORES. En estudios efectuados por los propios autores sobre el registro del discurso del paciente en las historias clínicas, el mismo apenas aparece lo cual establece la valoración que se le dá al mismo

3 NOTA DE LOS AUTORES. Quizás la adscripción de los hechos vitales al territorio hace que los geógrafos tengan un mejor abordaje en este sentido, ver: PUYOL, R, ESTEBANEZ, J.; MÉNDEZ, R "Geografia humana". Madrid. Ed. Cátedra. 1992.

4 HIPOCRATES. "De los aires, aguas o lugares" mencionado por Piedrola y cols en Medicina Preventiva y Salud Pública. ed. Salvat. Madrid, 1991

5 MENENDEZ, E. "Morir de alcohol". Alianza Editorial Mexicana. México. 1990

6 NOTA DE LOS AUTORES. Entendemos por PATOCENOSIS, aquel perfil de aconteceres mórbidos que afecta a una población, llevándola a la enfermedad y a la muerte 
suenan a estribillo final de ciertas explicaciones sobre lo que es o deben ser nuestras realidades ${ }^{7}$.

\section{MATERIAL, MÉTODOS Y RESULTADOS}

$\mathrm{Al}$ objeto de hacer pedagógicamente más inteligible esta presentación, describiremos las actividades realizadas de agrupándolas secuencialmente en fases:

2.1 DETERMINACIÓN DE LAS ENFERMEDADES A ESTUDIAR.

2.2 DETERMINACIÓN DE LAS ZONAS DEL MUNDO EN LA QUE SE PRODUCEN LAS 291 ENFERMEDADES ESTUDIADAS.

2.3 ANÁLISIS CUANTITATIVO (CIFRAS ABSOLUTAS) DE ENFERMEDADES QUE SE PRODUCEN EN CADA ZONA.

2.4 ANÁLISIS DE ENFERMEDADES ENDÉMICAS POR ZONAS.

2.5 ANÁLISIS DE LA SITUACIÓN DE LAS ENFERMEDADES CUYA INMUNIZACIÓN ESTÁ RECOMENDADA O ES OBLIGATORIA, EN LOS CALENDARIOS VACUNALES -EN CASI TODO EL MUNDO-, POR ZONAS.

\subsection{Determinación de las enfermedades a estudiar}

La patocenosis se refiere al perfil de morbilidad y mortalidad que afecta a una población, ${ }^{8}$. Nosotros hemos sesgado conscientemente el análisis, por decisión del grupo, decidiéndonos por estudiar la patocenosis de todas las áreas del globo, pero solamente los aspectos de morbilidad y, dado que, pretendiamos la mayor sensibilidad posible, preferimos escoger como objetivo a estudiar, las enfermedades transmisibles, ${ }^{10},{ }^{11},{ }^{12},{ }^{13}$, utilizando como nomenclator para las mismas, la lista CIE- $10^{14}$.

7 NOTA DE LOS AUTORES. Que a modo de letanias o tantras, desgranamos a veces, lenta y tediosamente en nuestras horas de docencia.

8 NOTA DE LOS AUTORES. O lo que es lo mismo de que enferma o muere una población

9 NOTA DE LOS AUTORES: Aceptamos la segunda hipótesis que indica Marcel Sendrail en "Historia cultural de la enfermedad". Madrid. Espasa Calpe.1983; de " la interdependencia entre las condiciones patológicas y las condiciones generales de la civilización : por un lado, las enfermedades dependen de los mismos factores geográficos y climáticos que las civilizaciones, por otro cada civilización debido a sus costumbres, sus leyes, sus principios, se crea una patología que le es propia... Una sociedad escoge sus enfermedades, orienta su destino patológico".

10 NOTA DE LOS AUTORES. Entre las patologías de tipo transmisible y las degenerativas, como resultado de una Técnica de Grupo Nominal, se planteó la conveniencia de utilizar las primeras, por que nos podian dar una información más sensible al comparar la patocenosis existente en las diferentes zonas del mundo a estudiar

11 BENENSON, A. "Manual para el control de las enfermedades transmisibles". Washington Organización Panamericana de la Salud. 1997 (Decimosexta edición).

12 ENFERMEDAD TRANSMISIBLE. Cualquier enfermedad causada por un agente infeccioso específico sus productos tóxicos, que se manifiesta por la transmisión del mismo agente o sus productos, de un animal o persona infectados o de un reservorio inanimado a un huésped susceptible, de forma directa o indirecta.

13 NOTA DE LOS AUTORES. Hemos analizado 291 enfermedades transmisibles.

14 NOTA DE LOS AUTORES. Hemos elegido la Clasificación Internacional de Enfermedades $n^{\circ} 10$. 


\subsection{Análisis cuantitativo (cifras absolutas) de enfermedades que se producen en cada zona}

Compilamos la información, dándonos cuenta de que el número de enfermedades que afectaban a cada una de las zonas era similar, es decir, que las patocenosis de cada zona eran muy parecidas, aunque con significativas diferencias de todas las zonas con Europa (Gráfica y Tabla $n^{\circ} 1$ ).

Obviamente, las diferencias no respondian a nuestra pretensión de observar diferencias sensibles entre zonas, por ello nos propusimos refinar el análisis.

\subsection{Análisis de enfermedades endémicas por zonas}

Al objeto de perfeccionar la sensibilidad del análisis fijamos nuestra atención en las denominadas enfermedades endémicas ${ }^{17}$, y estudiamos solamente aquellas que se definian como tales (explícitamente) según el último informe de la Organización Panamericana de la Salud ${ }^{18}$.

Cuando redujimos nuestro punto de mira únicamente al análisis, de estas enfermedades, los resultados empezaron a tomar un cariz o un perfil diferente. Las zonas que, a nuestro entender, estaban "señaladas" por unos perfiles mórbidos más amplios, se correspondian con aquellas que nosotros esperábamos (Gráfica y Tabla $\mathrm{n}^{\mathfrak{0}} 2$ ).

\subsection{Análisis de la situación de las enfermedades cuya inmunización está recomendada o es obligatoria, en los calendarios vacunales -en casi todo el mundo-, por zonas}

Para completar el recorrido por el "bosque", nos decidimos a estudiar que pasaba con aquellas enfermedades transmisibles susceptibles de inmunización, es decir que pueden ser afrontadas con unos costes limitados con respecto a otro tipo de técnicas, y con unos resultados más que espectaculares cuando se realiza sistemáticamente y con buenas condiciones técnicas (preparaciones, cadena del frío...).

Elegimos como enfermedades objetivo: difteria, tétanos, tosferina, hepatitis B, poliomielitis virica, sarampión. parotiditis, y rubéola; comparando tipos de países ${ }^{19}$, los desarrollados y aquellos que podíamos denominar subdesarrollados o en vías de desarrollo (Tabla $n^{\circ} 3$ ), encontrando unas diferencias más que notables.

\section{CONCLUSIONES}

$1^{\text {a }}$. La visión que planteamos habitualmente, pedagógicamente hablando, impide a los estudiantes percibir claramente los problemas del complejo salud-enfermedad-sistema de cuidados, en torno a la patocenosis de las poblaciones.

\footnotetext{
17 ENDEMIA. Presencia continua de una enfermedad o agente infeccioso en una zona geográfica determinada; ver BENENSON, A. "Manual para el control de las enfermedades transmisibles". op. cit.

18 BENENSON, A. “ Manual para el control de las enfermedades transmisibles" op. cit

19 NOTA DE LOS AUTORES. Categorías utilizadas en el propio informe de la OPS, ya mencionado.
} 
$2^{\mathrm{a}}$. El analizar las enfermedades, no como entidades individuales, sino como procesos que afectan a poblaciones concretas, permite a los estudiantes y profesionales del dominio de la salud, adquirir una postura crílica.

$3^{\text {a }}$. El análisis de todas las enfermedades Iransmisibles en cuanto a casos y zonas en que se dan, enmascara, en principio, una realidad debido a ciertos efectos de los movimientos de la población (turismo, guerras, desplazamientos,...) que permiten la aparición de casos en zonas, donde estas enfermedades, no son un problema de salud pública, de primer orden.

$4^{\mathrm{a}}$. Cuando analizamos las poblaciones que viven en territorios endémicos de enfermedades, los resultados nos trasladan a los problemas que subyacen: pobreza, falta de sanidad pública o de acceso a ella...

$5^{\mathrm{a}}$. Cuando pretendemos evidenciar esle hecho, comparar el efecto de ciertas enfermedades para las que hay medios más que eficaces de prevención, entre países desarrollados y no desarrollados, nos deja la evidencia de que la salud es casi traducible a Don Dinero

\section{BIBLIOGRAFÍA}

BENENSON, A. Manual para el control de las enfermedades transmisibles. Washington Organización Panamericana de la Salud. 1997 (Decimosexta edición).

BENENSON, A. Mamual para el control de las enfermedades transmisibles. Washington Organización Panamericana de la Salud. 1993 (Decimoquinta edición).

MAZARRASSA, L. y COLS, Salud Píblica y Enfermeria Comunitaria. Madrid. Ed Mc Graw Hill. 1996

PIEDROLA GIL, G., Medicina preventiva y social. Madrid. Ed. Salvat. 1996 (Octava edición).

PIEDROLA GIL, G., Medicina preventiva y social. Madrid. Ed. Salvat. 1991 (Novena edición).

HIPOCRATES. "De los aires, aguas o lugares" mencionado por Piedrola y cols en Medicina Preventiva y Sahud Piblica. Ed. Salval. Madrid, 1991.

JENICEK, M.; CLÉROUX, R., Epidemiología.Principios-lécnicas-aplicaciones. Edit. Salvat. Barcelona, 1988

MENÉNDEZ, E., Morir de alcohol. Alianza Editorial Mexicana. México. 1990

PUYOL, R.; ESTEBANEZ, J.; MÉNDEZ, R., Geografia humana, Madrid. Ed. Cátedra. 1992.

DONATI, P., Manual de Sociologia de la Salud. Madrid. Ed. Diaz de Santos S.A. 1987

MARTíN ZURRO, A. Y COLS., Atención Primaria. Barcelona. Ed Mosby-Doyma Libros. 1994

SENDRAIL, M., Historia cultural de la enfermedad. Madrid. Ed. Espasa Calpe 1983 


\section{TABLAS Y GRÁFICAS.}

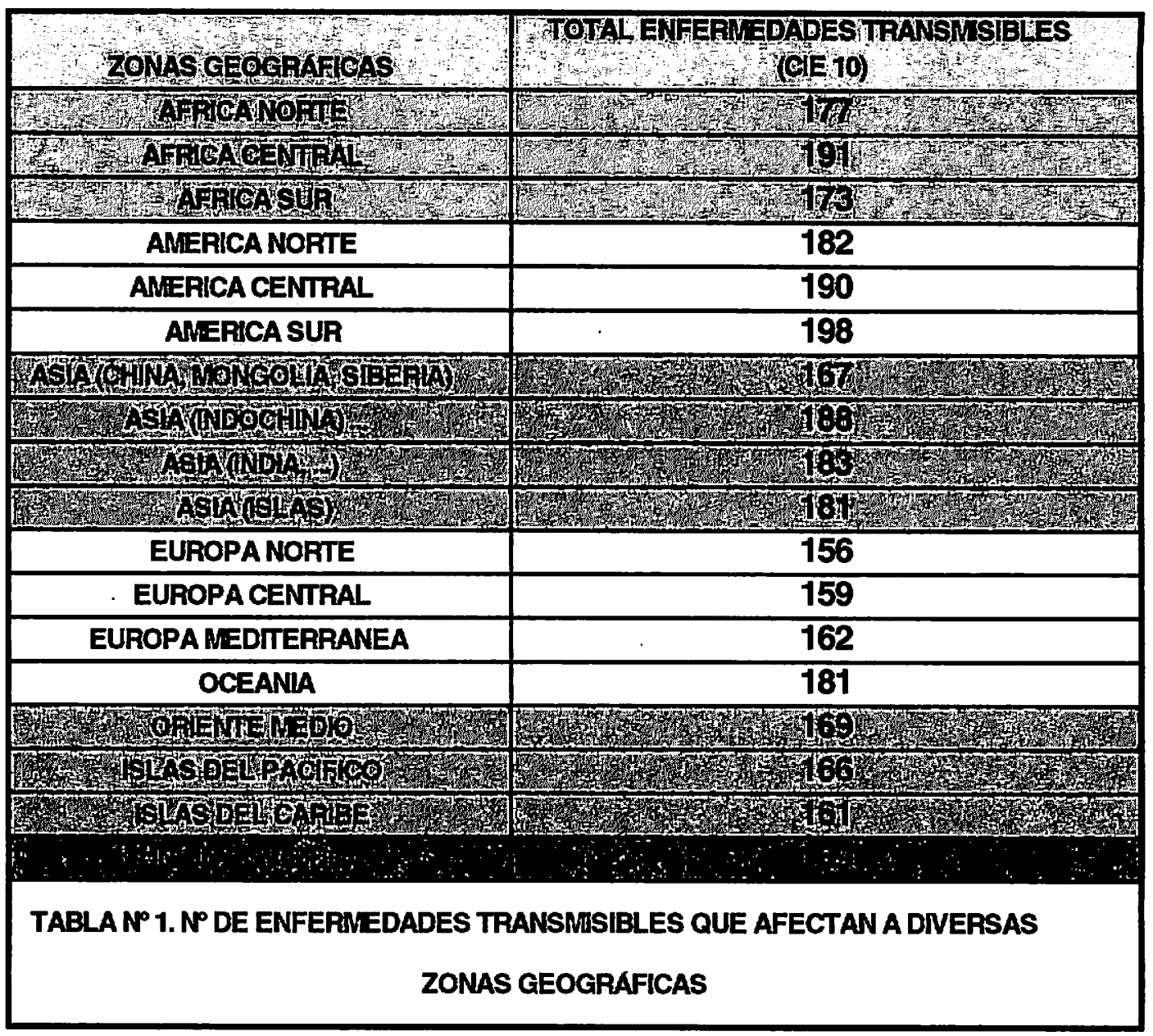


GRÁFICA NP. NUMERO DE ENFERMEDADES TRANSMASIBLES QUE AFECTAN A CIERTAS ZONAS DEL MUNDO (SOBRE LAS 291 ANALIZADAS)

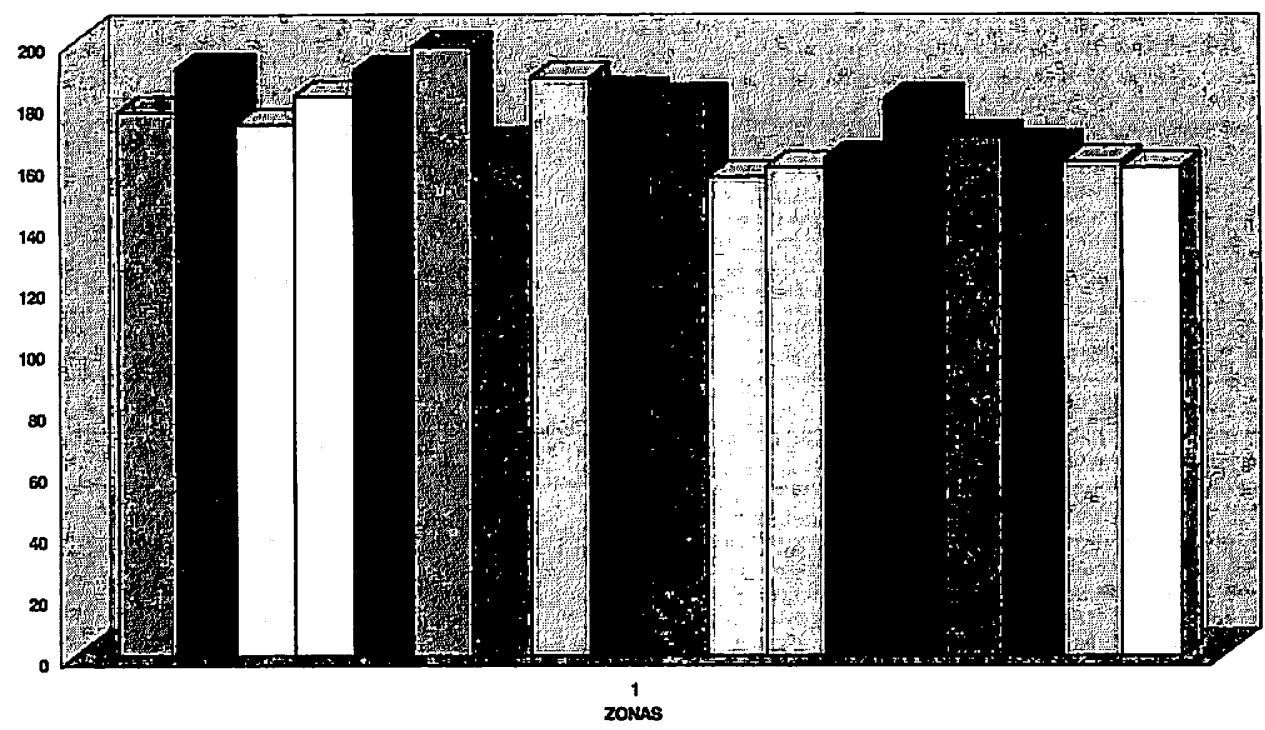

Q AFRECA NORTE

I AMERICA CENIRAL

- ASIA (NDIA, ...)

DEUFOPA MEDTERPANEA

- ISLAS DEL CARBE
- AFHICA CENTRAL

פAMERICA SUR

vasia (ISLAS)

- OCEANAa

qUNONSOVETICA
DAFRICA SUR

口 ASIA (CAINA, MONGOLLA, SIBERLA)

DEUROPANORTE

IORAENTE MEDNO
DAMERTCA NORTE

DASIA (NDOCOINA) ... DEUPOPA CENTRAL UISLAS DEL PACHICO

\begin{tabular}{|c|c|}
\hline ZONAS GEOGRAFICAS & $\begin{array}{l}\text { TOTAL ENFERMEDADES TRANSMMSIBLES (CIE 10) } \\
\text { POR ZONAS ENDÉMACAS }\end{array}$ \\
\hline ATHONORTE & 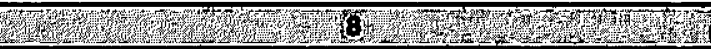 \\
\hline 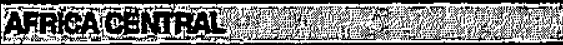 & 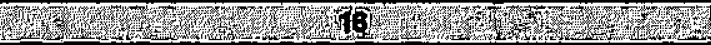 \\
\hline AFICASH & W \\
\hline AMERICA NORTE & 5 \\
\hline ANEERICA CENTRAL & 12 \\
\hline AMERICA SUR & 14 \\
\hline 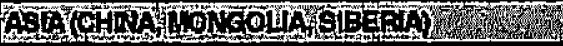 & $t_{2}+2$ \\
\hline 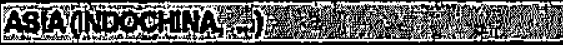 & Nin \\
\hline 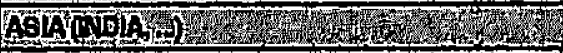 & (6) \\
\hline 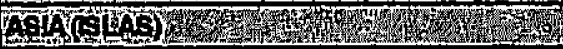 & 20 \\
\hline EUROPANORTE & 1 \\
\hline EUROPA CENTRAL & 2 \\
\hline EUROPA MEDITERRANEA & 4 \\
\hline OCEANIA & 8 \\
\hline 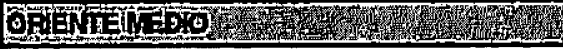 & 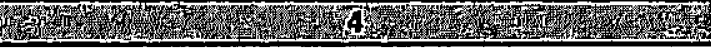 \\
\hline ISLAS DEL PACFFICO & 7 \\
\hline ISLAS DEL CAPIBE & 8 \\
\hline 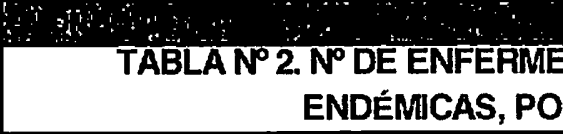 & ÁREAS GEOGRÁFICAS \\
\hline
\end{tabular}




\section{GRÁFICA No 2 NP DE ENFERMEDADES TRANSMISIBLES QUE SÓN ENDÉMICAS , POR ÁREAS GEOGRÁFICAS}

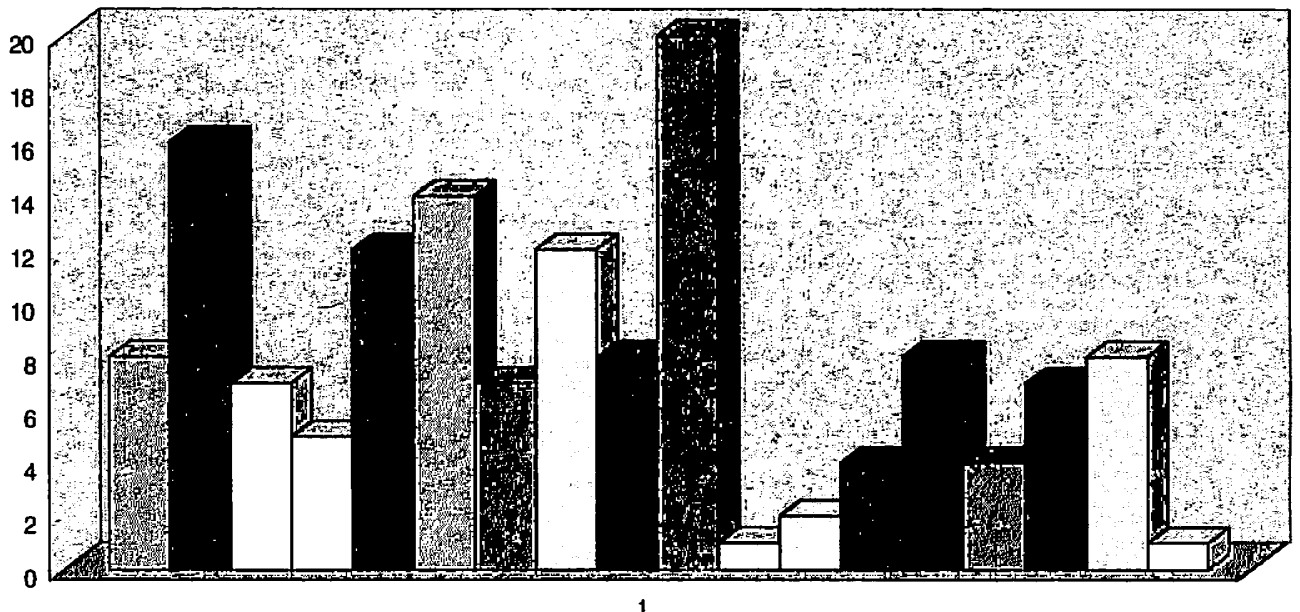

פAFRICA NORTE

口AMERICA NORTE

口 ASIA (CHINA, MONGOUA, SIBERIA)

口ASIA (ISLAS)

- EUROPA MEDITERRANEA

- ISLAS DEL PACIFICO
I AFRICA CENTRAL

- ANERICA CENTRAL

$\square A S I A$ (NDOCHINA, ...)

口EUROPA NORTE

- OCEANIA

口ISLAS DEL CARIBE
口AFRICA SUA

口 AMERICA SUR

- ASIA (INDIA, ...)

口EUROPA CENTRAL

๑ ORIENTE MEDIO

$\square$ UNION SOVIETICA

\begin{tabular}{|c|c|c|c|c|c|}
\hline & \multirow[b]{2}{*}{ PAISES DESARAOLLADOS } & \multicolumn{4}{|c|}{ PASES SUBDESARAOLLADOS } \\
\hline & & ABIA & Africh & $\begin{array}{c}\text { AHITACA CEKTRAL } \\
\text { Y DEL SUR } \\
\end{array}$ & $\begin{array}{l}\text { ANmGUA } \\
\text { UNoCN } \\
\text { SOVIIICA }\end{array}$ \\
\hline TETANOOS & Pococotivis & 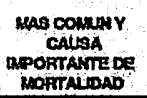 & 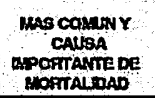 & 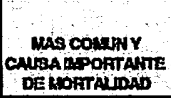 & \\
\hline TOSFERANA & $\begin{array}{c}\text { HA DISMMUUTDO } \\
\text { ECTRAORDINARIAMENTE (1) }\end{array}$ & 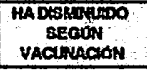 & 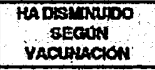 & 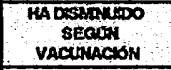 & \\
\hline DAFTERAA & POCO COIAUN & $\therefore$ & 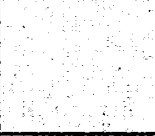 & 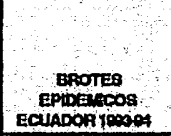 & 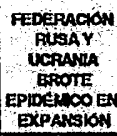 \\
\hline KEPATIIIS B & PREVALENCLA DE UN $5 \%$ & 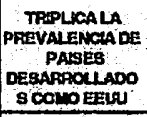 & 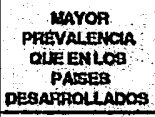 & $\begin{array}{l}\text { MAYOA } \\
\text { PFEVALENCIA OLE } \\
\text { ENLOSOS PAISES } \\
\text { DEQANAOLADOS }\end{array}$ & \\
\hline VIRUS POL.ONMELTTS & A PLANTO DE SER EFRADICADA & 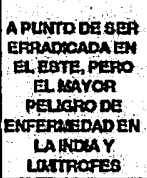 & 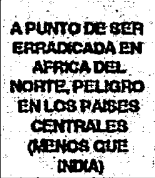 & & \\
\hline SARAMPION & $\begin{array}{l}\text { HA DISNONUIDO EN AL, GUMOS } \\
\text { PAISES HASTA EL } 9 \%\end{array}$ & 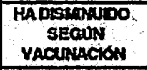 & 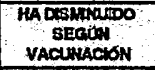 & 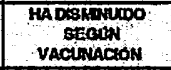 & \\
\hline PAPOTDTTS & $\begin{array}{c}\text { HA DISHNUTDO } \\
\text { EXTALOOPONARAANENTE (1) }\end{array}$ & 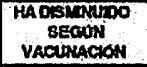 & 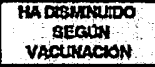 & 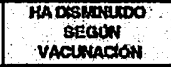 & \\
\hline FUBEOLA & $\begin{array}{l}\text { HA DISMERUIDO } \\
\text { EXTRAOFDINAFAATENTE }\end{array}$ & 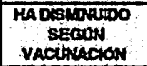 & 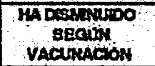 & 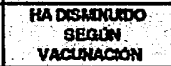 & \\
\hline TABLANי3. APROXINAC & $\begin{array}{l}\text { LEMAENEASE ALADISTF } \\
\text { ESTAN EN LOS CALEN }\end{array}$ & $\begin{array}{l}\text { CONDE LASE } \\
\text { IOS VACUNAL }\end{array}$ & FFERIVEDADES & UE GENERICAME & \\
\hline
\end{tabular}

